\title{
Photoswitchable dismantlers of biomaterials made of amyloid fibrils.
}

\section{Przemyslaw Kozminski ${ }^{1}$, Krzysztof Lyczko ${ }^{1}$, Zuzana Bednarikova ${ }^{2}$, Zuzana Gazova ${ }^{2}$, Imola Wilhelm ${ }^{3}$, Istvan Krizbai ${ }^{3}$, Grzegorz Wieczorek ${ }^{4}$, Dorota Niedzialek ${ }^{4}$}

\author{
${ }^{l}$ Institute of Nuclear Chemistry and Technology, Warsaw, Poland; \\ ${ }^{2}$ Institute of Experimental Physics, Slovak Academy of Sciences, Kosice, Slovakia; \\ ${ }^{3}$ nstitute of Biophysics, Biological Research Center, Hungarian Academy of Sciences, Szeged, Hungary; \\ ${ }^{4}$ Institute of Biochemistry and Biophysics Polish Academy of Sciences, Warsaw, Poland;
}

$$
\text { dniedzialek@ibb.waw.pl }
$$

Azobenzene molecules exhibit reversible light-triggered changes in geometrical structure (cis/trans isomerism) and nanoscale mechanical properties. For this reason, they have have been already widely used in materials science to build photo-mechanical responsive systems, incorporated in electronic switchable devices, used for graphene slicing, optical switching and data storage. We have been exploring further the photoresponsive nature of newly designed azobenzene derivatives and exploiting the potential of these smart materials for the generation of novel low-cost relevant molecular machines for biotechnology enabling the control of production/degradation of amyloid-based biomaterials. To this end, we have carefully functionalized azobenzene molecules by properties-by-design approach supported by the state-of-the-art in silico molecular design techniques as well as structure determination by X-ray crystallography. According to our Thioflavin-T Assay and NMR experimental results, the custom-designed azobenzene switches interact with the amyloid assemblies and intercalate between their strands. Stimulation with light, by inducing conformational change of azobenzene molecules, puts mechanical stress on the amyloid strands, eventually dissociating them and, in turn liquidizing the amyloid fibrils. Our in vitro studies of the designed azobenzene derivatives indicate no evidence of their cytotoxicity. Hence, it should be possible, in general, to use them for photo-control amyloid degradation in living systems, which constitutes a big encouragement for designing new azobenzene derivatives for biomedical applications, for example novel therapies against severe infections caused by amyloid biofilm forming bacteria or amyloid associated neurogenerative conditions such as Alzheimer's disease.

Keywords: stimuli responsive materials, photoswitches, controlled degradation of biomaterials 